\title{
On the Existence and Uniqueness of Solutions for Multidimensional Fractional Stochastic Differential Equations with Variable Order
}

\author{
Seyfeddine Moualkia ${ }^{1,2}$ and Yong $\mathrm{Xu}^{1, *(D)}$ \\ 1 School of Mathematics and Statistics, Northwestern Polytechnical University, Xi'an 710072, China; \\ 15596656563@163.com \\ 2 Department of Mathematics, 8 Mai 1945-Guelma University, Guelma 24000, Algeria \\ * Correspondence: hsux3@nwpu.edu.cn
}

Citation: Moualkia, S.; Xu, Y. On the Existence and Uniqueness of

Solutions for Multidimensional Fractional Stochastic Differential Equations with Variable Order. Mathematics 2021, 9, 2106. https:/ / doi.org/10.3390/math9172106

Academic Editor: Emanuela Rosazza Gianin

Received: 29 July 2021

Accepted: 25 August 2021

Published: 31 August 2021

Publisher's Note: MDPI stays neutral with regard to jurisdictional claims in published maps and institutional affiliations.

Copyright: (c) 2021 by the authors. Licensee MDPI, Basel, Switzerland. This article is an open access article distributed under the terms and conditions of the Creative Commons Attribution (CC BY) license (https:/ / creativecommons.org/licenses/by/ $4.0 /)$.

\begin{abstract}
Fractional stochastic differential equations are still in their infancy. Based on some existing results, the main difficulties here are how to deal with those equations if the fractional order is varying with time and how to confirm the existence of their solutions in this case. This paper is about the existence and uniqueness of solutions to the fractional stochastic differential equations with variable order. We prove the existence by using the Picard iterations and propose new sufficient conditions for the uniqueness.
\end{abstract}

Keywords: fractional stochastic differential equations; variable order; existence and uniqueness

\section{Introduction}

This work is concerned with the existence and uniqueness of solutions to the following problem of $k$-dimensional nonlinear fractional stochastic differential equations with variable order (VOFSDEs)

$$
D^{\alpha(t)} x(t, \omega)=f_{1}(t, x(t, \omega), \omega)+f_{2}(t, x(t, \omega), \omega) \frac{d W(t)}{d t}, t \in J=[0, T], \omega \in \Omega,
$$

where $T>0, f_{1}:[0, T] \times C_{b} \times \Omega \rightarrow \mathbb{R}^{k}$ and $f_{2}:[0, T] \times C_{b} \times \Omega \rightarrow \mathbb{R}^{k \times k^{\prime}}$ are given functions, $W(t)$ is a $k^{\prime}$-dimensional standard Brownian motion on a complete probability space $(\Omega, \mathcal{F}, \mathbb{P})$ with a filtration $\left\{\mathcal{F}_{t}\right\}_{0 \leq t \leq T}$ which increasing and right-continuous while $\mathcal{F}_{0}$ consists of all $\mathbb{P}$-null sets, $x(0, \omega)=0$ and $D^{\alpha(t)}$ is the Caputo fractional derivative of variable order $\alpha(t)$.

Fractional calculus is a generalization of traditional integer-order integration and differentiation actions onto non-integer order. The fundamental properties of the fractional differential system or its structure are always time-varying, such as time-varying coefficients, variable-order exponents, etc. Fractional differential equations with variable order are still at an early stage of development. They have attracted many researchers' attention due to its numerous applications in various branches of science and engineering, such as fluid mechanics [1] dynamics [2,3], diffusion [4], and so on.

On the other hand, stochastic differential equations (SDEs) are considered an effective tool in the description of many processes and systems in different fields. Several authors [5-8] have dealt with different research interests for classical SDEs. Then, they extended their studies to the fractional case (FSDEs with constant order $\alpha$ ) and investigated many results like existence, uniqueness, and stability for various classes of FSDEs (see [9-16]).

While most of the above results of existence and uniqueness for stochastic differential equations have been shown in the constant fractional order case, there is real need to pose an important question: how to deal with those equations if the fractional order is varying 
with time? and how to confirm the existence of their solutions in this case? Motivated by these facts, our purpose is to develop the classical SDEs towards fractional stochastic differential equations involving variable order $\alpha(t)$. In particular, we aim to extend and improve the existence and uniqueness results that appeared in $[14,16]$.

In this paper, we introduce a new class of Caputo-type nonlinear VOFSDEs (see Equation (1)). To treat that, we mainly establish a new set of sufficient conditions for nonlinear functions which generalizes the ones assumed in $[14,16]$. Then, we construct an iteration sequence involving variable fractional order $\alpha(t)$, which differs from the ones defined in $[14,16]$. After that, based on our analysis and discussion, we prove that the considered sequence is converging under those conditions to the unique solution of our studied problem (1). Consequently, we get a significant update in the stochastic theory, it is the existence and uniqueness of solutions of VOFSDEs (1), which contributes to the derivation of new results of optimal control and filtering of fractional stochastic dynamical systems. In addition, we consider the exact solution and the same analogue of these results to solve the exact controllability of VOFSDEs (1).

\section{Preliminaries}

In this section, we introduce some definitions and preliminary facts that we need in proving of our results, which can be found in $[2,3]$

Definition 1. The Riemann-Liouville fractional integral of order $\alpha(t)$ for function $f$ is defined as follows

$$
I^{\alpha(t)} f(t)=\int_{a}^{t} \frac{(t-s)^{\alpha(s)-1}}{\Gamma(\alpha(s))} f(s) d s, \quad t>a .
$$

Definition 2. The Caputo fractional derivative of order $\alpha(t)$ for function $f$ is defined for any $t>a$ as follows

$$
D^{\alpha(t)} f(t)=\left\{\begin{array}{l}
\int_{a}^{t} \frac{(t-s)^{-\alpha(s)}}{\Gamma(1-\alpha(s))} f^{\prime}(s) d s, \quad 0<\alpha(t)<1, \\
f(t), \quad \alpha(t)=0
\end{array}\right.
$$

where $\Gamma$ denotes the Gamma function.

Now, we define the following notations:

$\mathbb{R}^{k}$ and $\mathbb{R}_{+}$denote the $k$-dimensional Euclidean space and the set of all nonnegative real numbers, respectively. Let $L^{2}\left(\Omega, \mathbb{R}^{k}\right)$ be the space of all random functions $G_{1}(t, \omega)$ defined on a complete probability space $(\Omega, \mathcal{F}, \mathbb{P})$ into $\mathbb{R}^{k}$, such that $\mathbb{E}\left(\int_{0}^{T}\left|G_{1}(t, \omega)\right|^{2} d t\right)<\infty$, where $\mathbb{E}$ denotes the expected value of the random process. Hereafter, $C_{b}:=C\left([0, T], L^{2}\left(\Omega, \mathbb{R}^{k}\right)\right)$ be the space of all continuous and bounded functions $G_{2}(t, \omega)$ defined on $[0, T]$ into $L^{2}\left(\Omega, \mathbb{R}^{k}\right)$, such that $G_{2}(t, \omega)$ is $\mathcal{F}_{t}$-measurable for each $t \in[0, T]$. Consider $C_{b}$ endowed with the maximum norm.

Now, we make the following assumptions:

Assumption 1. The functions $f_{1}(t, x, \omega)$ and $f_{2}(t, x, \omega)$ are jointly measurable for any $x \in C_{b}$, and continuous for all $t \in J$ and a.e. $\omega \in \Omega$, with values in $L^{2}\left(\Omega, \mathbb{R}^{k}\right)$;

Assumption 2. $\alpha(t)$ is a continuous measurable function concerning $t \in \mathbb{R}_{+}$, and bounded between its minimal and maximal values as follows $1 / 2<\alpha_{*} \leq \alpha(t) \leq \alpha^{*}<1$;

Assumption 3. There exist bounded and continuous functions $N_{1}, N_{2}: J \rightarrow \mathbb{R}_{+}$, such that

$$
\mathbb{E}\left|f_{1}(t, x, \omega)\right|^{2} \leq N_{1}(t)\left(1+\mathbb{E}|x|^{2}\right), \text { and } \mathbb{E}\left|f_{2}(t, x, \omega)\right|^{2} \leq N_{2}(t)\left(1+\mathbb{E}|x|^{2}\right),
$$

for every $(t, x) \in J \times C_{b}$ and for a.e. $\omega \in \Omega$. For the sake of simplicity, we assume that the functions $N_{1}(t)$ and $N_{2}(t)$ have the same upper bound $N^{*}$; 
Assumption 4. $f_{1}(t, x, \omega)$ and $f_{2}(t, x, \omega)$ satisfy the following condition for all $x_{1}, x_{2} \in C_{b}$

$$
\begin{aligned}
\left|f_{1}\left(t, x_{2}, \omega\right)-f_{1}\left(t, x_{1}, \omega\right)\right|^{2} & \leq \ell_{1}(t, \omega)\left|x_{2}-x_{1}\right|^{2}, \\
\text { and }\left|f_{2}\left(t, x_{2}, \omega\right)-f_{2}\left(t, x_{1}, \omega\right)\right|^{2} & \leq \ell_{2}(t, \omega)\left|x_{2}-x_{1}\right|^{2},
\end{aligned}
$$

where $\ell_{1}(\cdot, \cdot)$ and $\ell_{2}(\cdot, \cdot)$ are nondecreasing bounded continuous functions from $J \times \Omega$ into $\mathbb{R}_{+}$;

Assumption 5. There exists a random linear positive bounded operator $\Psi$ defined on $\Omega \times C_{b}$ such that $\lim _{n \rightarrow \infty}\left\|\Psi^{n}(\omega)\right\|^{\frac{1}{n}}<1$ and

$$
\mathbb{E}\left|\Phi(\omega) x_{2}-\Phi(\omega) x_{1}\right|^{2} \leq \Psi(\omega) \mathbb{E}\left|x_{2}-x_{1}\right|^{2}, \text { for } x_{1}, x_{2} \in C_{b}
$$

where $\Phi$ is a random continuous operator defined on $\Omega \times C_{b}$;

If the functions $\alpha(\cdot), N_{i}(\cdot)$, and $\ell_{i}(\cdot, \cdot)$ are constants, then these special cases have been considered in papers $[14,16]$ (see also paper [10]).

Definition 3. A function $x(t, \omega)$ is called a random solution to the problem (1), if $x(t, \omega) \in C_{b}$ and satisfies the following integral equation

$$
x(t, \omega)=\int_{0}^{t} \frac{(t-s)^{\alpha(s)-1}}{\Gamma(\alpha(s))} f_{1}(s, x(s, \omega), \omega) d s+\int_{0}^{t} \frac{(t-s)^{\alpha(s)-1}}{\Gamma(\alpha(s))} f_{2}(s, x(s, \omega), \omega) d W(s),
$$

for all $t \in[0, T]$ and for a.e. $\omega \in \Omega$.

Lemma 1 ([17]). Suppose that $a \geq 0, b>0$, and $v_{1}(t)$ and $v_{2}(t)$ are nonnegative function locally integrable on $0 \leq t \leq T$ with

$$
v_{2}(t) \leq v_{1}(t)+a \int_{0}^{t}(t-s)^{b-1} v_{2}(s) d s
$$

Then,

$$
v_{2}(t) \leq v_{1}(t)+\int_{0}^{t} \sum_{j=1}^{\infty} \frac{(a \Gamma(b))^{j}}{\Gamma(j b)}(t-s)^{j b-1} v_{1}(s) d s, \quad 0 \leq t \leq T
$$

\section{Main Results}

In this section, we shall discuss the existence and uniqueness of solutions to the VOFSDEs (1).

Theorem 1. Assume that Assumptions 1-3 hold, then the problem (1) has at least one solution in $C_{b}$.

Proof. Let us define the following Picard sequence $\left\{x_{n}\right\}_{n \geq 0}$ on $[0, T]$ with $x_{0}(0, \omega)=0$

$$
\begin{aligned}
x_{n}(t, \omega) & =\int_{0}^{t} \frac{(t-s)^{\alpha(s)-1}}{\Gamma(\alpha(s))} f_{1}\left(s, x_{n-1}(s, \omega), \omega\right) d s \\
& +\int_{0}^{t} \frac{(t-s)^{\alpha(s)-1}}{\Gamma(\alpha(s))} f_{2}\left(s, x_{n-1}(s, \omega), \omega\right) d W(s), \quad n \geq 1
\end{aligned}
$$

for all $t \in[0, T]$ and a.e. $\omega \in \Omega$. 
Suppose that $\lambda>1$. Thanks to the Cauchy-Schwartz inequality, Itô's isometry and Assumption 3, we obtain

$$
\begin{aligned}
\mathbb{E}\left|x_{n}(t, \omega)\right|^{2} \leq & 2 \mathbb{E}\left|\int_{0}^{t} \frac{(t-s)^{\alpha(s)-1}}{\Gamma(\alpha(s))} f_{1}\left(s, x_{n-1}(s, \omega), \omega\right) d s\right|^{2} \\
& +2 \mathbb{E}\left|\int_{0}^{t} \frac{(t-s)^{\alpha(s)-1}}{\Gamma(\alpha(s))} f_{2}\left(s, x_{n-1}(s, \omega), \omega\right) d W(s)\right|^{2}, \\
\leq & 2 T \int_{0}^{t}\left(\frac{(t-s)^{\alpha(s)-1}}{\Gamma(\alpha(s))}\right)^{2} \mathbb{E}\left|f_{1}\left(s, x_{n-1}(s, \omega), \omega\right)\right|^{2} d s \\
& +2 \int_{0}^{t}\left(\frac{(t-s)^{\alpha(s)-1}}{\Gamma(\alpha(s))}\right)^{2} \mathbb{E}\left|f_{2}\left(s, x_{n-1}(s, \omega), \omega\right)\right|^{2} d s, \\
\leq & 2 T N^{*} \int_{0}^{t}\left(\lambda \cdot \frac{t-s}{\lambda}\right)^{2 \alpha(s)-2}\left(1+\mathbb{E}\left|x_{n-1}(s, \omega)\right|^{2}\right) d s \\
& +2 N^{*} \int_{0}^{t}\left(\lambda \cdot \frac{t-s}{\lambda}\right)^{2 \alpha(s)-2}\left(1+\mathbb{E}\left|x_{n-1}(s, \omega)\right|^{2}\right) d s, \\
\leq & 2 T N^{*} \lambda^{2-2 \alpha_{*}} \int_{0}^{t}(t-s)^{2 \alpha_{*}-2}\left(1+\mathbb{E}\left|x_{n-1}(s, \omega)\right|^{2}\right) d s \\
& +2 N^{*} \lambda^{2-2 \alpha_{*}} \int_{0}^{t}(t-s)^{2 \alpha_{*}-2}\left(1+\mathbb{E}\left|x_{n-1}(s, \omega)\right|^{2}\right) d s, \\
\leq & 2(T+1) N^{*} \lambda^{2-2 \alpha_{*}}\left[\frac{T^{2 \alpha_{*}-1}}{2 \alpha_{*}-1}+\int_{0}^{t}(t-s)^{2 \alpha_{*}-2} \mathbb{E}\left|x_{n-1}(s, \omega)\right|^{2} d s\right], \\
\leq & k_{1}+k_{2} \int_{0}^{t}(t-s)^{2 \alpha_{*}-2} \mathbb{E}\left|x_{n-1}(s, \omega)\right|^{2} d s,
\end{aligned}
$$

where $k_{1}=\frac{2(T+1) N^{*} \lambda^{2-2 \alpha_{*}} T^{2 \alpha_{*}-1}}{2 \alpha_{*}-1}$ and $k_{2}=2(T+1) N^{*} \lambda^{2-2 \alpha_{*}}$.

On the other hand, for any $i \geq 1$, it is clear that

$$
\max _{1 \leq n \leq i} \mathbb{E}\left|x_{n-1}(s, \omega)\right|^{2} \leq \max _{1 \leq n \leq i} \mathbb{E}\left|x_{n}(s, \omega)\right|^{2}
$$

Therefore,

$$
\max _{1 \leq n \leq i} \mathbb{E}\left|x_{n}(t, \omega)\right|^{2} \leq k_{1}+k_{2} \int_{0}^{t}(t-s)^{2 \alpha_{*}-1-1} \max _{1 \leq n \leq i} \mathbb{E}\left|x_{n}(s, \omega)\right|^{2} d s .
$$

By Lemma 1, we have

$$
\begin{aligned}
\max _{1 \leq n \leq i} \mathbb{E}\left|x_{n}(t, \omega)\right|^{2} & \leq k_{1}\left(1+\int_{0}^{t} \sum_{j=1}^{\infty} \frac{\left(k_{2} \Gamma\left(2 \alpha_{*}-1\right)\right)^{j}}{\Gamma\left(j\left(2 \alpha_{*}-1\right)\right)}(t-s)^{j\left(2 \alpha_{*}-1\right)-1} d s\right) \\
& \leq k_{1}\left(1+\sum_{j=1}^{\infty} \frac{\left(k_{2} \Gamma\left(2 \alpha_{*}-1\right) T^{2 \alpha_{*}-1}\right)^{j}}{\Gamma\left(j\left(2 \alpha_{*}-1\right)+1\right)}\right) \\
& \leq k_{1}\left(1+E_{2 \alpha_{*}-1,1}\left(k_{2} \Gamma\left(2 \alpha_{*}-1\right) T^{2 \alpha_{*}-1}\right)<\infty,\right.
\end{aligned}
$$

where $E_{2 \alpha_{*}-1,1}(\cdot)$ is the Mittag-Leffler function which can be found in [17]. Because $i$ is arbitray, we get $\mathbb{E}\left|x_{n}(t, \omega)\right|^{2}<\infty$, which proves the boundedness of $\left\{x_{n}\right\}_{n \geq 0}$.

By repeating a similar above process, the case of $0<\lambda \leq 1$ can be obtained easily without multiplying or dividing the term $(t-s)$ by $\lambda$. 
Now, because $f_{1}$ and $f_{2}$ are a functions in $L^{2}\left(\Omega, \mathbb{R}^{k}\right)$, the following integrals

$$
I_{1}=\int_{0}^{t} f_{1}\left(s, x_{n-1}(s, \omega), \omega\right) d s, \text { and } I_{2}=\int_{0}^{t} f_{2}\left(s, x_{n-1}(s, \omega), \omega\right) d W(s),
$$

exist on $[0, T]$, and represent the Lebesgue's integral and the Itô's stochastic integral, respectively. Because the assumption on $\alpha(s)$, it is obvious that the kernel $\frac{(t-s)^{\alpha(s)-1}}{\Gamma(\alpha(s))}$ is bounded. In addition, according to Assumption 3, it then follows

$$
\begin{aligned}
& \int_{0}^{t} \mathbb{E}\left|\frac{(t-s)^{\alpha(s)-1}}{\Gamma(\alpha(s))} f_{1}\left(s, x_{n-1}(s, \omega), \omega\right)\right|^{2} d s<\infty, \\
& \int_{0}^{t} \mathbb{E}\left|\frac{(t-s)^{\alpha(s)-1}}{\Gamma(\alpha(s))} f_{2}\left(s, x_{n-1}(s, \omega), \omega\right)\right|^{2} d s<\infty,
\end{aligned}
$$

which implies that the integrals

$$
\begin{aligned}
& I_{11}=\int_{0}^{t} \frac{(t-s)^{\alpha(s)-1}}{\Gamma(\alpha(s))} f_{1}\left(s, x_{n-1}(s, \omega), \omega\right) d s, \quad \text { and } \\
& I_{22}=\int_{0}^{t} \frac{(t-s)^{\alpha(s)-1}}{\Gamma(\alpha(s))} f_{2}\left(s, x_{n-1}(s, \omega), \omega\right) d W(s),
\end{aligned}
$$

are well defined. In view of integrals $I_{11}, I_{22}$ and Equation (2), we deduce that the sequence $\left\{x_{n}\right\}_{n \geq 0}$ is well defined on $[0, T]$.

According to Assumption 1, the maps $\omega \mapsto f_{1}\left(t, x_{n-1}(t, \omega), \omega\right)$ and $\omega \mapsto f_{2}\left(t, x_{n-1}(t, \omega), \omega\right)$ are measurable for all $t \in[0, T]$. Also, according to Assumption 2 the products $\left(\frac{(t-s)^{\alpha(s)-1}}{\Gamma(\alpha(s))} f_{1}\left(s, x_{n-1}(s, \omega), \omega\right)\right)$ and $\left(\frac{(t-s)^{\alpha(s)-1}}{\Gamma(\alpha(s))} f_{2}\left(s, x_{n-1}(s, \omega), \omega\right)\right)$ of continuous and measurable functions are again measurable for all $t \in[0, T]$. In addition, the integral is the limit of the finite sum of measurable functions. So, the maps

$$
\begin{aligned}
\omega & \mapsto \int_{0}^{t} \frac{(t-s)^{\alpha(s)-1}}{\Gamma(\alpha(s))} f_{1}\left(s, x_{n-1}(s, \omega), \omega\right) d s, \quad \text { and } \\
\omega & \mapsto \int_{0}^{t} \frac{(t-s)^{\alpha(s)-1}}{\Gamma(\alpha(s))} f_{2}\left(s, x_{n-1}(s, \omega), \omega\right) d W(s),
\end{aligned}
$$

are measurable. In view of (2), we deduce that the sequence $\left\{x_{n}\right\}_{n \geq 0}$ is measurable for all $t \in[0, T]$.

Now, let $\epsilon>0$, if we choose $\delta, \delta_{1}>0$ such that $0<\left(T+\delta_{1}+2\right) \delta_{1}^{2 \alpha_{*}-1} \leq \delta$ with

$$
\delta \leq \frac{\epsilon\left(2 \alpha_{*}-1\right)}{4 N^{*} R \lambda^{2-2 \alpha_{*}}}, \quad \text { where } \lambda>1 \text {, and for a constant } R>0 .
$$


Then for $0 \leq t_{1}<t_{2} \leq T$ with $0<t_{2}-t_{1}<\delta_{1}$ and $0<t_{1}-s<t_{2}-s<\lambda$, using Cauchy-Schwartz inequality and Itô's isometry, we get

$$
\begin{aligned}
& \mathbb{E}\left|x_{n}\left(t_{2}, \omega\right)-x_{n}\left(t_{1}, \omega\right)\right|^{2} \\
& \leq 4 \mathbb{E}\left|\int_{0}^{t_{1}}\left(\frac{\left(t_{2}-s\right)^{\alpha(s)-1}}{\Gamma(\alpha(s))}-\frac{\left(t_{1}-s\right)^{\alpha(s)-1}}{\Gamma(\alpha(s))}\right) f_{1}\left(s, x_{n-1}(s, \omega), \omega\right) d s\right|^{2} \\
& +4 \mathbb{E}\left|\int_{t_{1}}^{t_{2}} \frac{\left(t_{2}-s\right)^{\alpha(s)-1}}{\Gamma(\alpha(s))} f_{1}\left(s, x_{n-1}(s, \omega), \omega\right) d s\right|^{2} \\
& +4 \mathbb{E}\left|\int_{t_{1}}^{t_{2}} \frac{\left(t_{2}-s\right)^{\alpha(s)-1}}{\Gamma(\alpha(s))} f_{2}\left(s, x_{n-1}(s, \omega), \omega\right) d W(s)\right|^{2} \\
& +4 \mathbb{E}\left|\int_{0}^{t_{1}}\left(\frac{\left(t_{2}-s\right)^{\alpha(s)-1}}{\Gamma(\alpha(s))}-\frac{\left(t_{1}-s\right)^{\alpha(s)-1}}{\Gamma(\alpha(s))}\right) f_{2}\left(s, x_{n-1}(s, \omega), \omega\right) d W(s)\right|^{2}, \\
& \leq 4 \int_{0}^{t_{1}}\left(\frac{\left(t_{2}-s\right)^{\alpha(s)-1}-\left(t_{1}-s\right)^{\alpha(s)-1}}{\Gamma(\alpha(s))}\right)^{2} d s \int_{0}^{t_{1}} \mathbb{E}\left|f_{1}\left(s, x_{n-1}(s, \omega), \omega\right)\right|^{2} d s \\
& +4 \int_{t_{1}}^{t_{2}}\left(\frac{\left(t_{2}-s\right)^{\alpha(s)-1}}{\Gamma(\alpha(s))}\right)^{2} d s \int_{t_{1}}^{t_{2}} \mathbb{E}\left|f_{1}\left(s, x_{n-1}(s, \omega), \omega\right)\right|^{2} d s \\
& +4 \int_{t_{1}}^{t_{2}}\left(\frac{\left(t_{2}-s\right)^{\alpha(s)-1}}{\Gamma(\alpha(s))}\right)^{2} \mathbb{E}\left|f_{2}\left(s, x_{n-1}(s, \omega), \omega\right)\right|^{2} d s \\
& +4 \int_{0}^{t_{1}}\left(\frac{\left(t_{2}-s\right)^{\alpha(s)-1}-\left(t_{1}-s\right)^{\alpha(s)-1}}{\Gamma(\alpha(s))}\right)^{2} \mathbb{E}\left|f_{2}\left(s, x_{n-1}(s, \omega), \omega\right)\right|^{2} d s, \\
& \leq 4 N^{*} \int_{0}^{t_{1}}\left(\left(t_{1}-s\right)^{2 \alpha(s)-2}-\left(t_{2}-s\right)^{2 \alpha(s)-2}\right) d s \int_{0}^{t_{1}}\left(1+\mathbb{E}\left|x_{n-1}(s, \omega)\right|^{2}\right) d s \\
& +4 N^{*} \int_{t_{1}}^{t_{2}}\left(t_{2}-s\right)^{2 \alpha(s)-2} d s \int_{t_{1}}^{t_{2}}\left(1+\mathbb{E}\left|x_{n-1}(s, \omega)\right|^{2}\right) d s \\
& +4 N^{*} \int_{t_{1}}^{t_{2}}\left(t_{2}-s\right)^{2 \alpha(s)-2}\left(1+\mathbb{E}\left|x_{n-1}(s, \omega)\right|^{2}\right) d s \\
& +4 N^{*} \int_{0}^{t_{1}}\left(\left(t_{1}-s\right)^{2 \alpha(s)-2}-\left(t_{2}-s\right)^{2 \alpha(s)-2}\right)\left(1+\mathbb{E}\left|x_{n-1}(s, \omega)\right|^{2}\right) d s, \\
& \leq 4 t_{1} N^{*} R \int_{0}^{t_{1}}\left(\left(\lambda \cdot \frac{t_{1}-s}{\lambda}\right)^{2 \alpha(s)-2}-\left(\lambda \cdot \frac{t_{2}-s}{\lambda}\right)^{2 \alpha(s)-2}\right) d s \\
& +4\left(t_{2}-t_{1}\right) N^{*} R \int_{t_{1}}^{t_{2}}\left(\lambda \cdot \frac{t_{2}-s}{\lambda}\right)^{2 \alpha(s)-2} d s+4 N^{*} R \int_{t_{1}}^{t_{2}}\left(\lambda \cdot \frac{t_{2}-s}{\lambda}\right)^{2 \alpha(s)-2} d s \\
& +4 N^{*} R \int_{0}^{t_{1}}\left(\left(\lambda \cdot \frac{t_{1}-s}{\lambda}\right)^{2 \alpha(s)-2}-\left(\lambda \cdot \frac{t_{2}-s}{\lambda}\right)^{2 \alpha(s)-2}\right) d s, \\
& \leq 4 t_{1} N^{*} R \lambda^{2-2 \alpha_{*}} \int_{0}^{t_{1}}\left(\left(t_{1}-s\right)^{2 \alpha_{*}-2}-\left(t_{2}-s\right)^{2 \alpha_{*}-2}\right) d s \\
& +4\left(t_{2}-t_{1}\right) N^{*} R \lambda^{2-2 \alpha_{*}} \int_{t_{1}}^{t_{2}}\left(t_{2}-s\right)^{2 \alpha_{*}-2} d s+4 N^{*} R \lambda^{2-2 \alpha_{*}} \int_{t_{1}}^{t_{2}}\left(t_{2}-s\right)^{2 \alpha_{*}-2} d s \\
& +4 N^{*} R \lambda^{2-2 \alpha_{*}} \int_{0}^{t_{1}}\left(\left(t_{1}-s\right)^{2 \alpha_{*}-2}-\left(t_{2}-s\right)^{2 \alpha_{*}-2}\right) d s, \\
& \leq \frac{4 N^{*} R \lambda^{2-2 \alpha_{*}}}{2 \alpha_{*}-1}\left(T+\delta_{1}+2\right) \delta_{1}^{2 \alpha_{*}-1} \leq \frac{4 N^{*} R \lambda^{2-2 \alpha_{*}}}{2 \alpha_{*}-1} \delta .
\end{aligned}
$$


According to the relations (3) and (4), we get $\mathbb{E}\left|x_{n}\left(t_{2}, \omega\right)-x_{n}\left(t_{1}, \omega\right)\right|^{2} \leq \epsilon$, which means that $\left\{x_{n}\right\}_{n \geq 0}$ is equicontinuous. For the case where $0<\lambda \leq 1$, the steps of the proof rest similar, but $\delta$ will satisfy the condition $\delta \leq \frac{\epsilon\left(2 \alpha_{*}-1\right)}{4 N^{*} R}$.

Since the sequence $\left\{x_{n}\right\}_{n>0}$ is equicontinuous and uniformly bounded, the AscoliArzela's theorem assures that $\left\{x_{n}\right\}_{n>0}$ is a compact subset of $C_{b}$. We recall that $C_{b}$ is the space of continuous, bounded and $\overline{\mathcal{F}}_{t}$-measurable functions. It is a separable complete metric space with the metric $d$ defined by $d(x, y)=\max _{t \in[0, T]}\|x(t)-y(t)\|$ for $x, y \in C_{b}$.

Let $M^{2}\left(\Omega, C_{b}\right)$ be the space of $C_{b}$-valued random variables. Hence $\left\{x_{n}\right\}_{n>0} \subseteq M^{2}\left(\Omega, C_{b}\right)$. Recall that $\left\{x_{n}\right\}_{n>0}$ is bounded for all $t \in[0, T]$. Now by Prohorov's theorem, $\left\{x_{n}\right\}_{n>0}$ is totally $D$-bounded in $M^{2}\left(\Omega, C_{b}\right)$. Thus (see [18]), there exists a $D$-Cauchy subsequence $\left\{x_{n_{m}}\right\}$ of $\left\{x_{n}\right\}_{n \geq 0}$. Let us denote $\left\{x_{n_{m}}\right\}$ by $\left\{x_{m}\right\}$. By Skorokhod's theorem (see [19]), we can construct a sequence $\left\{Y_{m}\right\} \in M^{2}\left(\Omega, C_{b}\right)$ and a random variable $x \in M^{2}\left(\Omega, C_{b}\right)$ such that the distance

$$
\begin{gathered}
D\left(Y_{m}, x_{m}\right)=0, \quad \text { for } \quad m=1,2,3, \cdots \\
\mathbb{P}\left\{Y_{m} \rightarrow x\right\}=1, \quad \text { as } \quad m \rightarrow \infty .
\end{gathered}
$$

It is obvious that $x(t, \omega)$ is continuous and $\mathcal{F}_{t}$-measurable on $[0, T]$. Notice that $D\left(Y_{m}, x_{m}\right)=0$ means that $\left\{Y_{m}\right\}$ and $\left\{x_{m}\right\}$ have the same distribution. Hence $\left\{Y_{m}\right\}$ is bounded, so also $x(t, \omega)$ is bounded w.p.1 in view of (6).

Now, for all $t \in[0, T]$, we shall prove that the sequence $\left\{Y_{m}\right\}$ converges to the solution $x(t, \omega)$ of problem (1) w.p.1.

$$
\begin{aligned}
& \mathbb{E} \mid \int_{0}^{t} \frac{(t-s)^{\alpha(s)-1}}{\Gamma(\alpha(s))}\left(f_{1}\left(s, Y_{m}(s, \omega), \omega\right)-f_{1}(s, x(s, \omega), \omega)\right) d s \\
& +\left.\int_{0}^{t} \frac{(t-s)^{\alpha(s)-1}}{\Gamma(\alpha(s))}\left(f_{2}\left(s, Y_{m}(s, \omega), \omega\right)-f_{2}(s, x(s, \omega), \omega)\right) d W(s)\right|^{2} \\
\leq & 2 \int_{0}^{t}(t-s)^{2 \alpha(s)-2} d s \int_{0}^{t} \mathbb{E}\left|f_{1}\left(s, Y_{m}(s, \omega), \omega\right)-f_{1}(s, x(s, \omega), \omega)\right|^{2} d s \\
& +2 \int_{0}^{t}(t-s)^{2 \alpha(s)-2} \mathbb{E}\left|f_{2}\left(s, Y_{m}(s, \omega), \omega\right)-f_{2}(s, x(s, \omega), \omega)\right|^{2} d s, \\
\leq & \frac{2 \lambda^{2-2 \alpha_{*}} t^{2 \alpha_{*}-1}}{2 \alpha_{*}-1} \int_{0}^{t} \mathbb{E}\left|f_{1}\left(s, Y_{m}(s, \omega), \omega\right)-f_{1}(s, x(s, \omega), \omega)\right|^{2} d s \\
& +2 \lambda^{2-2 \alpha_{*}} \int_{0}^{t}(t-s)^{2 \alpha_{*}-2} \mathbb{E}\left|f_{2}\left(s, Y_{m}(s, \omega), \omega\right)-f_{2}(s, x(s, \omega), \omega)\right|^{2} d s, \\
\leq & \frac{2 \lambda^{2-2 \alpha_{*}} T^{2 \alpha_{*}-1}}{2 \alpha_{*}-1} \int_{0}^{T} \mathbb{E}\left|f_{1}\left(s, Y_{m}(s, \omega), \omega\right)-f_{1}(s, x(s, \omega), \omega)\right|^{2} d s \\
& +2 \lambda^{2-2 \alpha_{*}} \int_{0}^{T}(T-s)^{2 \alpha_{*}-2} \mathbb{E}\left|f_{2}\left(s, Y_{m}(s, \omega), \omega\right)-f_{2}(s, x(s, \omega), \omega)\right|^{2} d s .
\end{aligned}
$$

In view of Assumption 3, we have $\mathbb{E}\left|f_{1}\left(s, Y_{m}(s, \omega), \omega\right)\right|^{2} \leq N_{1}(s)\left(1+\mathbb{E}\left|Y_{m}(s, \omega)\right|^{2}\right)$ and $\mathbb{E}\left|f_{2}\left(s, Y_{m}(s, \omega), \omega\right)\right|^{2} \leq N_{2}(s)\left(1+\mathbb{E}\left|Y_{m}(s, \omega)\right|^{2}\right)$, for all $s \in[0, T]$. Since $f_{1}$ and $f_{2}$ are continuous in $x$, it follows that for any $\epsilon>0$, there exists a integer $j \geq 0$ such that

$\mathbb{E}\left|f_{1}\left(s, Y_{m}(s, \omega), \omega\right)-f_{1}(s, x(s, \omega), \omega)\right|^{2}<\epsilon / 2$, and

$\mathbb{E}\left|f_{2}\left(s, Y_{m}(s, \omega), \omega\right)-f_{2}(s, x(s, \omega), \omega)\right|^{2}<\epsilon / 2$, for all $m>j$.

Therefore,

$$
\begin{aligned}
\int_{0}^{T} \mathbb{E}\left|f_{1}\left(s, Y_{m}(s, \omega), \omega\right)-f_{1}(s, x(s, \omega), \omega)\right|^{2} d s & <\frac{\epsilon}{2} T \\
\int_{0}^{T}(T-s)^{2 \alpha_{*}-2} \mathbb{E}\left|f_{2}\left(s, Y_{m}(s, \omega), \omega\right)-f_{2}(s, x(s, \omega), \omega)\right|^{2} d s & <\frac{\epsilon}{2} \frac{T^{2 \alpha_{*}-1}}{\left(2 \alpha_{*}-1\right)} .
\end{aligned}
$$


Hence, for all $t \in[0, T]$ and a.e. $\omega \in \Omega$, we have

$$
\begin{aligned}
\int_{0}^{t} \frac{(t-s)^{\alpha(s)-1}}{\Gamma(\alpha(s))} f_{1}\left(s, Y_{m}(s, \omega), \omega\right) d s & \rightarrow \int_{0}^{t} \frac{(t-s)^{\alpha(s)-1}}{\Gamma(\alpha(s))} f_{1}(s, x(s, \omega), \omega) d s, \\
\int_{0}^{t} \frac{(t-s)^{\alpha(s)-1}}{\Gamma(\alpha(s))} f_{2}\left(s, Y_{m}(s, \omega), \omega\right) d W(s) & \rightarrow \int_{0}^{t} \frac{(t-s)^{\alpha(s)-1}}{\Gamma(\alpha(s))} f_{2}(s, x(s, \omega), \omega) d W(s), \\
\text { for all } m>j . &
\end{aligned}
$$

From Equations (2) and (5) and continuity of functions, we get

$$
Y_{m}(t, \omega)=\int_{0}^{t} \frac{(t-s)^{\alpha(s)-1}}{\Gamma(\alpha(s))} f_{1}\left(s, Y_{m}(s, \omega), \omega\right) d s+\int_{0}^{t} \frac{(t-s)^{\alpha(s)-1}}{\Gamma(\alpha(s))} f_{2}\left(s, Y_{m}(s, \omega), \omega\right) d W(s) .
$$

Relations (6)-(9) show that, by letting $m \rightarrow \infty$

$$
x(t, \omega)=\int_{0}^{t} \frac{(t-s)^{\alpha(s)-1}}{\Gamma(\alpha(s))} f_{1}(s, x(s, \omega), \omega) d s+\int_{0}^{t} \frac{(t-s)^{\alpha(s)-1}}{\Gamma(\alpha(s))} f_{2}(s, x(s, \omega), \omega) d W(s) .
$$

Consequently, we conclude that $x(t, \omega)$ is the random solution to problem (1). Further, because the boundedness of $x(t, \omega)$, it is obvious that $\mathbb{E}\left(\int_{0}^{T}|x(t, \omega)|^{2} d t\right)<\infty$, which completes the proof.

Now, we shall give the main result that assures uniqueness of the solution to the problem (1).

Theorem 2. Assume that Assumptions 1-5 hold, then Equation (1) has a unique random solution $x(t, \omega) \in C_{b}$.

Proof. We consider the operator $\Phi: \Omega \times C_{b} \rightarrow C_{b}$ defined by

$$
\Phi(\omega) x(t, \omega)=\int_{0}^{t} \frac{(t-s)^{\alpha(s)-1}}{\Gamma(\alpha(s))} f_{1}(s, x(s, \omega), \omega) d s+\int_{0}^{t} \frac{(t-s)^{\alpha(s)-1}}{\Gamma(\alpha(s))} f_{2}(s, x(s, \omega), \omega) d W(s),
$$

for all $t \in[0, T]$ and a.e. $\omega \in \Omega$.

Suppose $\lambda>1$, for each $x(t, \omega) \in C_{b}$, and with a similar process in the proof of the boundedness of sequence $\left\{x_{n}\right\}_{n \geq 0}$ (see page 4), we deduce that $\Phi(\omega)$ is uniformly bounded and well defined operator.

Now, we will show that $\Phi$ is a random operator. It is obvious from Assumptions 1 and 2 that $\omega \mapsto f_{1}(t, x(t, \omega), \omega)$ and $\omega \mapsto f_{2}(t, x(t, \omega), \omega)$ are measurable for all $t \in[0, T]$. Also, the products $\left(\frac{(t-s)^{\alpha(s)-1}}{\Gamma(\alpha(s))} f_{1}(s, x(s, \omega), \omega)\right)$ and $\left(\frac{(t-s)^{\alpha(s)-1}}{\Gamma(\alpha(s))} f_{2}(s, x(s, \omega), \omega)\right)$ of a continuous and measurable functions are again measurable for all $t \in[0, T]$. Further, the integral is a limit of a finite sum of measurable functions. So, the maps

$\omega \mapsto \int_{0}^{t} \frac{(t-s)^{\alpha(s)-1}}{\Gamma(\alpha(s))} f_{1}(s, x(s, \omega), \omega) d s$, and $\omega \mapsto \int_{0}^{t} \frac{(t-s)^{\alpha(s)-1}}{\Gamma(\alpha(s))} f_{2}(s, x(s, \omega), \omega) d W(s)$,

are measurable. It follows that $\Phi$ is a random operator from $\Omega \times C_{b}$ into $C_{b}$.

For the proof of continuity of $\Phi$, we assume that there exists a sequence $\left\{x_{i}\right\}_{i \geq 0}$ such that $x_{i} \rightarrow x$ in $C_{b}$ as $i \rightarrow \infty$, then because the continuity of $f_{1}$ and $f_{2}$ in $x$, we have $f_{1}\left(s, x_{i}(s, \omega), \omega\right) \rightarrow f_{1}(s, x(s, \omega), \omega)$, and $f_{2}\left(s, x_{i}(s, \omega), \omega\right) \rightarrow f_{2}(s, x(s, \omega), \omega)$ as $i \rightarrow \infty$. 
So, for all $t \in[0, T]$, and by using Cauchy-Schwartz inequality and Itô's isometry, we get

$$
\begin{aligned}
& \mathbb{E}\left|\Phi(\omega) x_{i}-\Phi(\omega) x\right|^{2} \\
\leq & 2 \mathbb{E}\left|\int_{0}^{t} \frac{(t-s)^{\alpha(s)-1}}{\Gamma(\alpha(s))}\left(f_{1}\left(s, x_{i}(s, \omega), \omega\right)-f_{1}(s, x(s, \omega), \omega)\right) d s\right|^{2} \\
& +2 \mathbb{E}\left|\int_{0}^{t} \frac{(t-s)^{\alpha(s)-1}}{\Gamma(\alpha(s))}\left(f_{2}\left(s, x_{i}(s, \omega), \omega\right)-f_{2}(s, x(s, \omega), \omega)\right) d W(s)\right|^{2}, \\
\leq & 2 \int_{0}^{t}\left(\frac{(t-s)^{\alpha(s)-1}}{\Gamma(\alpha(s))}\right)^{2} d s \int_{0}^{t} \mathbb{E}\left|f_{1}\left(s, x_{i}(s, \omega), \omega\right)-f_{1}(s, x(s, \omega), \omega)\right|^{2} d s \\
& +2 \int_{0}^{t}\left(\frac{(t-s)^{\alpha(s)-1}}{\Gamma(\alpha(s))}\right)^{2} \mathbb{E}\left|f_{2}\left(s, x_{i}(s, \omega), \omega\right)-f_{2}(s, x(s, \omega), \omega)\right|^{2} d s .
\end{aligned}
$$

Thanks to the Lebesgue dominated convergence theorem, we obtain $\mathbb{E}\left|\Phi(\omega) x_{i}-\Phi(\omega) x\right|^{2} \rightarrow$ 0 as $i \rightarrow \infty$.

Now, consider there exist constants $c, c^{\prime}>0$ such that $\mathbb{E}\left(\ell_{1}(t, \omega)\right) \leq c$ and $\mathbb{E}\left(\ell_{2}(t, \omega)\right) \leq$ $c^{\prime}$. For any $x_{1}, x_{2} \in C_{b}$, using Itô's isometry, Hölder's inequality and Assumption 4 , we obtain

$$
\begin{aligned}
& \mathbb{E}\left|\Phi(\omega) x_{2}-\Phi(\omega) x_{1}\right|^{2} \\
\leq & 2 T \int_{0}^{t} \frac{(t-s)^{2 \alpha(s)-2}}{(\Gamma(\alpha(s)))^{2}} \mathbb{E}\left|f_{1}\left(s, x_{2}(s, \omega), \omega\right)-f_{1}\left(s, x_{1}(s, \omega), \omega\right)\right|^{2} d s \\
& +2 \int_{0}^{t} \frac{(t-s)^{2 \alpha(s)-2}}{(\Gamma(\alpha(s)))^{2}} \mathbb{E}\left|f_{2}\left(s, x_{2}(s, \omega), \omega\right)-f_{2}\left(s, x_{1}(s, \omega), \omega\right)\right|^{2} d s, \\
\leq & 2 T \int_{0}^{t}\left(\lambda \cdot \frac{t-s}{\lambda}\right)^{2 \alpha(s)-2} \mathbb{E}\left(\ell_{1}(s, \omega)\right) \mathbb{E}\left|x_{2}(s, \omega)-x_{1}(s, \omega)\right|^{2} d s \\
& +2 \int_{0}^{t}\left(\lambda \cdot \frac{t-s}{\lambda}\right)^{2 \alpha(s)-2} \mathbb{E}\left(\ell_{2}(s, \omega)\right) \mathbb{E}\left|x_{2}(s, \omega)-x_{1}(s, \omega)\right|^{2} d s, \\
\leq & 2 c T \lambda^{2-2 \alpha_{*}} \int_{0}^{t}(t-s)^{2 \alpha_{*}-2} \mathbb{E}\left|x_{2}(s, \omega)-x_{1}(s, \omega)\right|^{2} d s \\
& +2 c^{\prime} \lambda^{2-2 \alpha_{*}} \int_{0}^{t}(t-s)^{2 \alpha_{*}-2} \mathbb{E}\left|x_{2}(s, \omega)-x_{1}(s, \omega)\right|^{2} d s, \\
\leq & 2\left(c T+c^{\prime}\right) \lambda^{2-2 \alpha_{*}} \int_{0}^{t}(t-s)^{2 \alpha_{*}-2} \mathbb{E}\left|x_{2}(s, \omega)-x_{1}(s, \omega)\right|^{2} d s \\
\leq & \Psi(\omega) \mathbb{E}\left|x_{2}(t, \omega)-x_{1}(t, \omega)\right|^{2} .
\end{aligned}
$$

It is obvious that the following operator $\Psi$ is random linear positive bounded and defined on $\Omega \times C_{b}$ into $C_{b}$ as follows

$$
\Psi(\omega) \mathbb{E}(x)^{2}=\left\{\begin{array}{l}
2\left(c T+c^{\prime}\right) \lambda^{2-2 \alpha_{*}} \int_{0}^{t}(t-s)^{2 \alpha_{*}-2} \mathbb{E}(x(s, \omega))^{2} d s, \quad \lambda>1, \\
2\left(c T+c^{\prime}\right) \int_{0}^{t}(t-s)^{2 \alpha_{*}-2} \mathbb{E}(x(s, \omega))^{2} d s, \quad 0<\lambda \leq 1 .
\end{array}\right.
$$

Suppose that $\lambda>1$, yields

$$
\left\|\left(\Psi(\omega) \mathbb{E}(x)^{2}\right)(t)\right\| \leq \frac{2\left(c T+c^{\prime}\right) \lambda^{2-2 \alpha_{*}} t^{2 \alpha_{*}-1}}{2 \alpha_{*}-1}\left\|\mathbb{E}(x)^{2}\right\|,
$$


it follows that

$$
\begin{aligned}
\left\|\left(\Psi^{2}(\omega) \mathbb{E}(x)^{2}\right)(t)\right\| & \leq 2\left(c T+c^{\prime}\right) \lambda^{2-2 \alpha_{*}} \int_{0}^{t}(t-s)^{2 \alpha_{*}-2}\left\|\left(\Psi(\omega) \mathbb{E}(x)^{2}\right)(s)\right\| d s, \\
& \leq\left(2\left(c T+c^{\prime}\right) \lambda^{2-2 \alpha_{*}}\right)^{2} \frac{\left\|\mathbb{E}(x)^{2}\right\|}{2 \alpha_{*}-1} \int_{0}^{t}(t-s)^{2 \alpha_{*}-2} s^{2 \alpha_{*}-1} d s .
\end{aligned}
$$

Posing $I=\int_{0}^{t}(t-s)^{2 \alpha_{*}-2} s^{2 \alpha_{*}-1} d s$, and taking $s=t \theta$, we get

$I=\int_{0}^{1}(1-\theta)^{2 \alpha_{*}-2} t^{2 \alpha_{*}-2}(t \theta)^{2 \alpha_{*}-1} t d \theta=t^{4 \alpha_{*}-2} B\left(2 \alpha_{*}, 2 \alpha_{*}-1\right)=t^{4 \alpha_{*}-2} \frac{\Gamma\left(2 \alpha_{*}\right) \Gamma\left(2 \alpha_{*}-1\right)}{\Gamma\left(4 \alpha_{*}-1\right)}$,

where $B(\cdot, \cdot)$ is the Beta function. Substituting the obtained expression of $I$ in $(10)$, yields

$$
\left\|\left(\Psi^{2}(\omega) \mathbb{E}(x)^{2}\right)(t)\right\| \leq\left(2\left(c T+c^{\prime}\right) \lambda^{2-2 \alpha_{*}} t^{2 \alpha_{*}-1}\right)^{2} \frac{\Gamma\left(2 \alpha_{*}\right) \Gamma\left(2 \alpha_{*}-1\right)}{\left(2 \alpha_{*}-1\right) \Gamma\left(4 \alpha_{*}-1\right)}\left\|\mathbb{E}(x)^{2}\right\|, t \in[0, T] .
$$

Using mathematical induction for any natural number $n>1$, we obtain

$$
\left\|\left(\Psi^{n}(\omega) \mathbb{E}(x)^{2}\right)(t)\right\| \leq\left(2\left(c T+c^{\prime}\right) \lambda^{2-2 \alpha_{*}} t^{2 \alpha_{*}-1} \Gamma\left(2 \alpha_{*}-1\right)\right)^{n} \frac{1}{\Gamma\left(n\left(2 \alpha_{*}-1\right)+1\right)}\left\|\mathbb{E}(x)^{2}\right\| .
$$

Therefore

$$
\begin{aligned}
\left\|\left(\Psi^{n}(\omega) \mathbb{E}(x)^{2}\right)\right\| & =\max _{0 \leq t \leq T}\left\|\left(\Psi^{n}(\omega) \mathbb{E}(x)^{2}\right)(t)\right\| \\
& \leq\left(2\left(c T+c^{\prime}\right) \lambda^{2-2 \alpha_{*}} T^{2 \alpha_{*}-1} \Gamma\left(2 \alpha_{*}-1\right)\right)^{n} \frac{1}{\Gamma\left(n\left(2 \alpha_{*}-1\right)+1\right)}\left\|\mathbb{E}(x)^{2}\right\| .
\end{aligned}
$$

Thus

$$
\left\|\Psi^{n}(\omega)\right\| \leq\left(2\left(c T+c^{\prime}\right) \lambda^{2-2 \alpha_{*}} T^{2 \alpha_{*}-1} \Gamma\left(2 \alpha_{*}-1\right)\right)^{n} \frac{1}{\Gamma\left(n\left(2 \alpha_{*}-1\right)+1\right)} .
$$

Taking $N=n\left(2 \alpha_{*}-1\right)$, we note that $N \rightarrow \infty$ as $n \rightarrow \infty$. Using an important property of Gamma function which generalizes the factorial, i.e., $\Gamma(N+1)=N$ ! for $N=0,1,2, \cdots$

Hence, for a.e. $\omega \in \Omega$ we deduce that: $\lim _{n \rightarrow \infty}\left\|\Psi^{n}(\omega)\right\|^{\frac{1}{n}}=0<1$.

By repeating a similar above process, the case of $0<\lambda \leq 1$ can be obtained easily. Thus, we conclude that the random operator $\Phi(\omega)$ has a unique fixed point $x(t, \omega) \in C_{b}$ such that $\Phi(\omega) x(t, \omega)=x(t, \omega)$, which is in turn a unique random solution of problem (1). It completed the proof.

\section{Example}

In the following, we shall present an example to illustrate the effectiveness of our obtained results.

Let $\Omega=[0,1]$ with the usual $\sigma$-algebra consisting of Lebesgue measurable subsets of $[0,1]$. Given a measurable function $x \in C_{b}\left([0,3000], L^{2}(\Omega, \mathbb{R})\right)$. Considering the problem (1) of the variable order FSDE with the given functions $f_{1}(t, x(t, \omega), \omega)=\frac{t^{1 / 5} \omega x(t, \omega)}{1.5 \times 10^{10}\left(1+e^{-x(t, \omega)}\right)}$, $f_{2}(t, x(t, \omega), \omega)=\frac{\sqrt{\omega t} x(t, \omega)}{\left(1+4 e^{-x(t, \omega)}\right) 4 \times 10^{10}}, \alpha(t)=\frac{3}{5}+\frac{t}{8000}$, and $W(t)$ denotes a standard onedimensional Brownian motion defined on the probability space $(\Omega, \mathcal{F}, \mathbb{P})$ with filtration $\left\{\mathcal{F}_{t}\right\}$ for every $t \in[0,3000]$ and $\omega \in[0,1]$. So, the FSDE with variable order can be rewrite as follows

$$
D^{\left(\frac{3}{5}+\frac{t}{8000}\right)} x(t, \omega)=\frac{t^{1 / 5} \omega x(t, \omega)}{1.5 \times 10^{10}\left(1+e^{-x(t, \omega)}\right)}+\frac{\sqrt{\omega t} x(t, \omega)}{\left(1+4 e^{-x(t, \omega)}\right) 4 \times 10^{10}} \frac{d W(t)}{d t} .
$$


For each $t \in[0,3000]$, we have $\frac{1}{2}<\alpha_{*}=0.6 \leq \alpha(t) \leq \alpha^{*}=0.975<1, \mathbb{E}\left|f_{1}(t, x(t, \omega), \omega)\right|^{2}$ $\leq \frac{t^{2 / 5} \mu_{1} \mathbb{E}|x|^{2}}{2.25 \times 10^{20}} \leq \frac{t^{2 / 5} \mu_{1}}{2.25 \times 10^{20}}\left(1+\mathbb{E}|x|^{2}\right)=N_{1}(t)\left(1+\mathbb{E}|x|^{2}\right)$ and $\mathbb{E}\left|f_{2}(t, x(t, \omega), \omega)\right|^{2} \leq \frac{t \mu_{2} \mathbb{E}|x|^{2}}{16 \times 10^{20}} \leq$ $\frac{t \mu_{2}}{16 \times 10^{20}}\left(1+\mathbb{E}|x|^{2}\right)=N_{2}(t)\left(1+\mathbb{E}|x|^{2}\right)$, where $\mu_{1}=\mathbb{E}\left[\omega^{2}\right]<\infty$ and $\mu_{2}=\mathbb{E}[\omega]<\infty$. It is clear that the Assumptions 1-3 are satisfied. Hence, Theorem 1 guarantees that problem (11) has at least one solution $x(t, \omega) \in C_{b}$.

Now, for any $x_{1}, x_{2} \in C_{b}$, the functions $f_{1}$ and $f_{2}$ satisfy the following conditon

$$
\begin{aligned}
\left|f_{1}\left(t, x_{2}(t, \omega), \omega\right)-f_{1}\left(t, x_{1}(t, \omega), \omega\right)\right|^{2} & =\left|\frac{t^{1 / 5} \omega}{1.5 \times 10^{10}}\left(\frac{x_{2}(t, \omega)}{1+e^{-x_{2}(t, \omega)}}-\frac{x_{1}(t, \omega)}{1+e^{-x_{1}(t, \omega)}}\right)\right|^{2}, \\
& \leq \frac{t^{2 / 5} \omega^{2}}{2.25 \times 10^{20}}\left|x_{2}(t, \omega)-x_{1}(t, \omega)\right|^{2},
\end{aligned}
$$

and

$$
\begin{aligned}
\left|f_{2}\left(t, x_{2}(t, \omega), \omega\right)-f_{2}\left(t, x_{1}(t, \omega), \omega\right)\right|^{2} & =\left|\frac{\sqrt{\omega t}}{4 \times 10^{10}}\left(\frac{x_{2}(t, \omega)}{1+4 e^{-x_{2}(t, \omega)}}-\frac{x_{1}(t, \omega)}{1+4 e^{-x_{1}(t, \omega)}}\right)\right|^{2} \\
& \leq \frac{\omega t}{16 \times 10^{20}}\left|x_{2}(t, \omega)-x_{1}(t, \omega)\right|^{2}
\end{aligned}
$$

where $\ell_{1}(t, \omega)=\frac{t^{2 / 5} \omega^{2}}{2.25 \times 10^{20}}$ and $\ell_{2}(t, \omega)=\frac{\omega t}{16 \times 10^{20}}$. It implies that, the Assumption 4 is satisfied.

Now, according to the nature of functions $f_{1}, f_{2}$ and $\alpha(t)$, it is clear that the following operator

$$
(\Phi x)(t, \omega)=\int_{0}^{t} \frac{(t-s)^{\alpha(s)-1}}{\Gamma(\alpha(s))} f_{1}(s, x(s, \omega), \omega) d s+\int_{0}^{t} \frac{(t-s)^{\alpha(s)-1}}{\Gamma(\alpha(s))} f_{2}(s, x(s, \omega), \omega) d W(s),
$$

is random continuous bounded. Furthermore, taking $T=3000, c=\frac{10.93}{10^{20}}, c^{\prime}=\frac{187.5}{10^{20}}$, and $\lambda=20$, then we get

$$
\begin{aligned}
& \mathbb{E}\left|\Phi(\omega) x_{2}(t, \omega)-\Phi(\omega) x_{1}(t, \omega)\right|^{2} \\
\leq & \left(2\left(3000 \frac{10.93}{10^{20}}+\frac{187.5}{10^{20}}\right) 20^{0.8} \frac{3000^{0.2}}{0.2}\right) \mathbb{E}\left|x_{2}(t, \omega)-x_{1}(t, \omega)\right|^{2}, \\
\leq & 544.81\left(\frac{32790}{10^{20}}+\frac{187.5}{10^{20}}\right) \mathbb{E}\left|x_{2}(t, \omega)-x_{1}(t, \omega)\right|^{2}, \\
\leq & \frac{1.797}{10^{13}} \mathbb{E}\left|x_{2}(t, \omega)-x_{1}(t, \omega)\right|^{2} .
\end{aligned}
$$

Therefore, the Assumption 5 is satisfied. Hence, according to Theorem 2, we conclude that the operator $\Phi$ has a unique fixed point $x(t, \omega) \in C_{b}$, which is in turn a unique random solution of problem (11).

\section{Conclusions}

In this paper, we have obtained the existence and uniqueness of solutions for multidimensional fractional stochastic differential equations with variable order using Picard iterations and propose new sufficient conditions. In particular, we have introduced two extensions of the work in $[14,16]$, which are summarized as follows. The coefficients are random processes, and the fractional order $\alpha(t)$ is time-varying which has restricted between the minimal and maximal values i.e., $1 / 2<\alpha_{*} \leq \alpha(t) \leq \alpha^{*}<1$. We have defined an iteration sequence involving variable fractional order, which converges to the unique solution of the main problem. As an application, we have presented an example to show the benefit of the obtained results. If the fractional order in problem (1) is dependent on 
more than one variable, then the considered case can be taken as an open problem. This is what we desire to treat in future works.

Author Contributions: S.M.: Conceptualization, Investigation, Methodology \& writing the manuscript. Y.X.: Methodology, Supervision, Visualization, reviewing \& editing the manuscript. All authors have read and agreed to the published version of the manuscript.

Funding: This research received no external funding.

Conflicts of Interest: The authors declare that they have no competing interest.

\section{References}

1. Atangana, A.; Algahtani, R.T. Stability analysis of nonlinear thin viscous fluid sheet flow equation with local fractional variable order derivative. J. Comput. Theor. Nanosci. 2016, 13, 1-8. [CrossRef]

2. Zhang, S. The uniqueness result of solution to initial value problems of differential equations of variable-order. Rev. Real Acad. Cienc. Exactas Físicas Nat. Ser. A Matemáticas 2018, 112, 407-423. [CrossRef]

3. Zhang, S.; Hu, L. Unique existence result of approximate solution to initial value problem for fractional differential equation of variable order involving the derivative arguments on the half-axis. J. Math. 2019, 7, 1-23. [CrossRef]

4. Zheng, X.; Wang, H. An optimal-order numerical approximation to variable-order space-fractional diffusion equations on uniform or graded meshes. SIAM J. Numer. Anal. 2020, 58, 330-352. [CrossRef]

5. Mei, R.; Xu, Y.; Kurths, J. Transport and escape in a deformable channel driven by fractional Gaussian noise. Phys. Rev. E 2019, 100, 1-10. [CrossRef] [PubMed]

6. Han, M.; Xu, Y.; Pei, B. Mixed stochastic differential equations: Averaging principle result. Appl. Math. Lett. 2021, 112, 106705. [CrossRef]

7. Liu, Q.; Xu, Y.; Kurths, J. Bistability and stochastic jumps in an airfoil system with viscoelastic material property and random fluctuations. Comm. Nonlinear Sci. Numer. Simul. 2020, 84, 1-16. [CrossRef]

8. Baños, D.; Ortiz-Latorre, S.; Pilipenko, A.; Proske, F. Strong solutions of stochastic differential equations with generalized drift and multidimensional fractional Brownian initial noise. J. Theor. Prob. 2021. [CrossRef]

9. Chen, P.; Zhang, X.; Li, Y. Nonlocal problem for fractional stochastic evolution equations with solution operators. Fract. Calc. Appl. Anal. 2016, 6, 1507-1526. [CrossRef]

10. El-Borai, M.M.; El-Nadi, K.E.S.; Ahmed, H.M.; El-Owaidy, H.M.; Ghanem, A.S.; Sakthivel, R. Existence and stability for fractional parabolic integro-partial differential equations with fractional Brownian motion and nonlocal condition. Cogent Math. Stat. 2018, 5, 1460030. [CrossRef]

11. Xu, Y.; Zan, W.; Jia, W.; Kurths, J. Path integral solutions of the governing equation of SDEs excited by Lévy white noise. J. Comput. Phys. 2019, 394, 41-55. [CrossRef]

12. Wang, W.; Cheng, S.; Guo, Z.; Yan, X. A note on the continuity for Caputo fractional stochastic differential equations. Chaos Interdiscip. J. Nonlinear Sci. 2020, 30, 073106. [CrossRef] [PubMed]

13. Ramkumar, K.; Ravikumar, K.; Varshini, S. Fractional neutral stochastic differential equations with Caputo fractional derivative: Fractional Brownian motion, Poisson jumps, and optimal control. Stoch. Anal. Appl. 2020, 39, 157-176. [CrossRef]

14. Ahmadova, A.; Mahmudov, N. Existence and uniqueness results for a class of fractional stochastic neutral differential equations. Chaos Solitons Fractals 2020, 139, 110253. [CrossRef]

15. Pei, B.; Xu, Y.; Wu, J.L. Stochastic averaging for stochastic differential equations driven by fractional Brownian motion and standard Brownian motion. Appl. Math. Lett. 2020, 100, 106006. [CrossRef]

16. Guo, Z.; Hu, J.; Wang, W. Caratheodory's approximation for a type of Caputo fractional stochastic differential equations. Adv. Differ. Equ. 2020, 100,1-12. [CrossRef]

17. Wang, Y.; Xu, J.; Kloeden, P.E. Asymptotic behavior of stochastic lattice systems with a Caputo fractional time derivative. Nonlinear Anal. Theory Methods Appl. 2016, 135, 205-222. [CrossRef]

18. Prohorov, Y.V. Convergence of Random Processes and Limit Theorems in Probability Theory. Theory Probab. Its Appl. 1956, 1, 157-214. [CrossRef]

19. Billingsley, P. Weak Convergence of Measures: Application in Probability; CBMS-NSF Regional Conference Series in Applied Mathematics; SIAM: Philadelphia, PA, USA, 1971. 